\title{
MR Imaging of Prostate Cancer at 3T with an External Phased-Array Coil: Evaluation of T2-Weighted Images
}

\author{
JUN SAKODA*, MASAFUMI UCHIDA*, SHUJI NAGATA*, KEI MATSUOKA**, \\ TOSHI ABE* AND NAOFUMI HAYABUCHI* \\ Departments of Radiology* and Urology**, Kurume University School of Medicine, \\ Kurume 830-0011, Japan
}

Received 10 November 2009, accepted 25 December 2009

\begin{abstract}
Summary: 1.5T Magnetic resonance (MR) imaging has become an accepted method for assessing prostate cancer. However, the role of 1.5T MRI in local staging of prostate cancer is limited. It is hoped that 3.0T MRI will be more useful in local staging of prostate cancer. The purpose of this study was to evaluate the tissue contrast, artifact presence, and image quality of T2-weighted images (T2WI) of prostate cancer using 3T MRI with a phased-array coil at different slice thicknesses and fields of view (FOV). We examined 15 patients with prostate cancer. We obtained MR images at slice thicknesses of $2 \mathrm{~mm}$ and $5 \mathrm{~mm}$ in both small and large FOV. The image obtained at a slice thickness of $2 \mathrm{~mm}$ with a small FOV had inferior tissue contrast compared to the other images $(\mathrm{P}<0.05)$, but there was no statistically significant difference in contrast between images obtained at a slice thickness of $2 \mathrm{~mm}$ with a large FOV and those obtained at a slice thickness of $5 \mathrm{~mm}$. Artifacts were rated equally among the parameter combinations. The overall image quality obtained at a slice thickness of $2 \mathrm{~mm}$ with a large FOV was significantly superior to the other three imaging parameters $(\mathrm{p}<0.05)$. The image obtained at a slice thickness of $2 \mathrm{~mm}$ with a large FOV was superior to the other three images in evaluating T2WI of prostate cancer with 3T MRI.
\end{abstract}

Key words 3T MR-imaging, field of view (FOV), slice thickness, T2-weighted image, spatial resolution, prostate cancer

\section{INTRODUCTION}

Prostate cancer is the most common malignant tumor in men. Magnetic resonance (MR) imaging of the prostate has become an accepted method for staging prostate cancer. 1.5T MRI using an endorectal coil is currently the best imaging modality for the preoperative evaluation of prostate cancer.

For several years, 3T MRI has been used for research purposes, but it recently has become more widely used in the clinical setting. Because 3T MRI theoretically has a two-fold higher signal to noise rate
(SNR) compared to conventional 1.5T MRI, its application is expected to provide a more accurate characterization of various organs. For neurological applications it has been demonstrated that spatial resolution improves detail delineation and thus diagnostic accuracy [1-4]. Several studies reported that 3T MRI with a phased-array coil is equivalent to $1.5 \mathrm{~T}$ MRI with an endorectal coil in evaluating local staging accuracy for prostate cancer $[5,6]$. However, in practice, the increase in SNR of whole-body scanners with 3T MRI is not as straightforward as it may appear. 3T MRI needs to overcome several technical challenges in order to

Correspondence to: Kei Matsuoka, Department of Urology, Kurume University School of Medicine, 67 Asahi-machi, Kurume 830-0011, Japan. Tel: +81942-31-7576 Fax: +81-942-32-9405

Abbreviations: ADC, apparent diffusion coefficient; ASSET, array spatial sensitivity encoding technique; DWI, diffusion weighted image; EPI, echo planar image; ETL, echo train length; FOV, field of view; FSE, fast spin echo; MRI, magnetic resonance image; PSA, prostate-specific antigen; RF, radio frequency wave; ROI, region of interest; SAR, specific absorption rate; SNR, signal to noise rate; TE, echo time; T1WI, T1-weighted image; T2WI, T2weighted image; TR, repetition time; TRUS, transrectal ultrasound. 
achieve the expected improvements in image quality and SNR. The main challenge for the pulse sequence design at higher magnetic fields is increased radiofrequency energy deposition, which requires new strategies to reduce the specific absorption rate (SAR) [7]. Further technical challenges facing 3T MRI include increased $\mathrm{T} 1$ relaxation time [8], decreased $\mathrm{T} 2$ relaxation time $[9,10]$, insufficient radiofrequency power penetration [11], stronger susceptibility effects, and a larger chemical shift. Thus, 3T MRI examination of the prostate as part of the clinical routine has not been established as a standard procedure.

The purpose of this study was to evaluate the tissue contrast, artifact presence, and image quality of T2-weighted images (T2WI) of prostate cancer in different fields of view (FOV) and at different slice thicknesses, obtained using 3T MRI with a phased-array coil within clinically acceptable scan times.

\section{MATERIALS AND METHODS}

\section{Patients}

Because the purpose of this study was to evaluate the tissue contrast, artifact presence, and image quality of T2WI in prostate cancer, the specimens used covered all stages of prostate cancer. A total of 15 patients with prostate cancer verified by transrectal ultrasound (TRUS)-guided biopsy underwent 3.0T MRI using a surface coil. Patient age ranged from 56 to 79 years (median, 71 years). The median serum level of prostate-specific antigen (PSA) was $17.9 \mathrm{ng} / \mathrm{ml}$ (range, $4.0-368 \mathrm{ng} / \mathrm{ml}$ ), and the mean Gleason score was 7.8 (range, 6-10).

The study protocol was reviewed and approved by the ethics committee of Kurume University.

\section{Inclusion and exclusion criteria}

Patients with histologically confirmed prostate cancer without any contraindications for a MRI examination of the prostate (e.g., cardiac pacemaker, intracranial clip) were included in our study. Patients with previous surgical, hormonal, or radiation therapy, as well as patients with indistinct zonal anatomy or a biopsy-related hematoma caused by prostate cancer were excluded from the study.

\section{MR imaging technique}

We performed MR imaging using a 3T MR scanner (Signa HDx, GE Medical Systems, Milwaukee, WI, USA) with an 8-channel torso array coil in 2 patients and an 8-channel cardiac array coil in 13 patients. Patients were examined in the supine position. In all patients, we obtained fast spin echo (FSE) T2WI in the axial plane following a localizer scan. We used 4 different combinations of FOV and slice thickness. To reduce the SAR, T2WI were obtained by a new variable refocusing angle technique called tailored radio frequency wave (RF). We obtained T2WI with a small FOV $(18 \times 18 \mathrm{~cm})$ and a large FOV $(26 \times 26 \mathrm{~cm})$. We imaged $2 \mathrm{~mm}$ and $5 \mathrm{~mm}$ slices in both small and large FOV. Table 1 shows the imaging parameters used for the 4 different FOV/slice thickness combinations. The small FOV images were obtained with voxel sizes of $0.56 \times 1.13 \times 2 \mathrm{~mm}\left(1.27 \mathrm{~mm}^{3}\right)$ and $0.56 \times 1.13 \times 5$ $\mathrm{mm}\left(3.16 \mathrm{~mm}^{3}\right)$. The voxel sizes of the large FOV were $0.81 \times 1.02 \times 2 \mathrm{~mm}\left(1.65 \mathrm{~mm}^{3}\right)$ and $0.51 \times 0.81 \times 5$ $\mathrm{mm}\left(2.07 \mathrm{~mm}^{3}\right)$.

In addition, we obtained T1-weighted images (T1WI) and diffusion weighted images (DWI). We ob-

TABLE 1.

Imaging parameters used to obtain T2-weighted MR images of 4 FOV/slice thickness combinations

\begin{tabular}{lcccc}
\hline & $\begin{array}{c}\text { Small FOV } \\
\text { Thickness=2 mm }\end{array}$ & $\begin{array}{c}\text { Small FOV } \\
\text { Thickness=5 mm }\end{array}$ & $\begin{array}{c}\text { Large FOV } \\
\text { Thickness=2 mm }\end{array}$ & $\begin{array}{c}\text { Large FOV } \\
\text { Thickness=5 mm }\end{array}$ \\
\hline Acquisition time (min) & 4.13 & 3.58 & 3.58 & 3.20 \\
Measured voxel size (mm) & $0.56 / 1.13 / 2$ & $0.56 / 1.13 / 5$ & $0.81 / 1.02 / 2$ & $0.51 / 0.8 / 5$ \\
Parallel imaging & No & No & Yes $(2)$ & Yes $(2)$ \\
Band width (KHz) & \pm 50 & \pm 41.7 & \pm 50 & \pm 83.3 \\
TR/TE (ms) & $7667 / 102.1$ & $7200 / 99.9$ & $7200 / 104.2$ & $8000 / 100.6$ \\
Number of signal averaged & 4 & 4 & 4 & 3 \\
Echo train length & 20 & 20 & 20 & 20 \\
Matrix & $320 \times 16$ & $320 \times 160$ & $320 \times 256$ & $512 \times 320$ \\
Field of view $(\mathrm{cm})$ & $18 \times 18$ & $18 \times 18$ & $26 \times 26$ & $26 \times 26$ \\
\hline
\end{tabular}


tained FSE T1WI in the axial plane using the following parameters: FOV, $24 \times 24 \mathrm{~cm}$; slice thickness, 7 $\mathrm{mm}$; repetition time (TR)/echo time (TE) 675/7.5 ms; echo train length, $3 ; 320 \times 224$ matrix; interslice gap, 1 $\mathrm{mm}$. The axial DWI were obtained by single-shot echo planar imaging (EPI) using the following imaging parameters: FOV, $36 \times 36 \mathrm{~cm}$; slice thickness, $5 \mathrm{~mm}$; TR/ TE 4000-4500/83.6 ms, 320×256 matrix; average of 4 signals. Motion-probing gradients were applied in 3 directions to obtain DWI using diffusion gradients with $2 \mathrm{~b}$-values $\left(0\right.$ and $\left.1000 \mathrm{sec} / \mathrm{mm}^{2}\right)$. We used an arrey spatial sensitivity encoding technique (ASSET) factor of 2 .

To improve RF field inhomogeneity, a dielectric pad was positioned in front of the body. Before MR examination, 13 patients were given an intramuscular injection of $1 \mathrm{mg}$ glucagon (Glucagon G Novo; Novo Nordisk Pharma, Tokyo, Japan) to reduce bowel peristalsis. Glucagon was contraindicated in the remaining 2 patients, and we examined them without suppression of peristalsis.

\section{Image analysis}

On MR images, we considered any lesion fulfilling the following criteria to be prostate cancer: an area with (a) diffuse low signal intensity with mass effect or (b) circumscribed, round, or triangular-shaped localized hypointensity on T2WI and (c) reduced apparent diffusion coefficient (ADC) in comparison with the normal prostate. Locations of all suspected lesions identified by MR imaging were consistent with estimates obtained by TRUS-guided biopsy.

We reviewed the images using the Centricity RA 1000 picture archiving and communications system (PACS) available from GE Medical Systems.

First, to assess tissue contrasts (C) between muscle and prostate cancer we performed quantitative measurements of the tissue contrasts in regions of interest (ROI) according to the following relation: $\mathrm{C}=(\mathrm{A}-\mathrm{B}) /$ $(A+B)$, where $A$ and $B$ represent the signal intensities (SI) of tissues A and B, respectively. The ROI for the SI measurement was established in the largest possible area of prostate cancer (A), and then a ROI of similar size was placed in muscle (B) in the same side for each sequence. We assessed the tissue contrast as described in a previous study [12].

We assessed the presence of MR imaging artifacts separately, and assessed the following scores by consensus: no artifacts, 1 ; minor artifact, 2 ; moderate artifact (not diagnostically relevant), 3; strong artifact (diagnostically relevant), 4 ; and severe artifact (nondiagnostic study), 5 .
In addition, we evaluated the image quality of the seminal vesicle as well as overall image quality. Images were visually interpreted by two radiologists in consensus. Criteria for quality of the seminal vesicle images were margin, septation and subjective impression. Criteria for overall image quality were artifact, sharpness and overall subjective impression. The visual comparison of the images was subjectively classified as excellent, 1; good, 2; satisfactory, 3; moderate, 4 ; or poor, 5 .

\section{Statistical analysis}

Statistical analysis was performed with the data analysis program StatMate for Macintosh. For all tests, a $P$-value of less than 0.05 was considered to indicate a statistically significant difference.

We used one-way ANOVA to compare tissue contrasts between the 4 different parameter combinations. When there were statistically significant differences, we used the Newman-Keuls test to make paired comparisons between different imaging modes. We used the Kruskal-Wallis test to compare the image quality between the 4 parameter combinations. When there were statistically significant differences, we used the Dunn test to make paired comparisons between different imaging modes.

\section{RESULTS}

Tables 2 and 3 display results of the analyses comparing T2WI obtained for the 4 different FOV/slice thickness combinations.

Quantitative contrast analysis revealed that images obtained at a slice thickness of $2 \mathrm{~mm}$ with a small FOV were significantly inferior to those obtained at a slice thickness of $5 \mathrm{~mm}$ with the same FOV $(P<0.05)$ and both slice thicknesses with the large FOV $(P<0.01)$. Despite this, there was no statistically significant difference between the images obtained at slice thicknesses of $2 \mathrm{~mm}$ and $5 \mathrm{~mm}$ with a large FOV (Table 2).

TABLE 2.

Contrast of image by parameter combinations

\begin{tabular}{|c|c|c|}
\hline Group & Contrast & \\
\hline Small FOV with slice thickness $=2 \mathrm{~mm}$ & $0.32 \pm 0.05]_{*}-$ & \\
\hline Small FOV with slice thickness $=5 \mathrm{~mm}$ & $0.37 \pm 0.06^{*} \mid * *$ & \\
\hline Large FOV with slice thickness $=2 \mathrm{~mm}$ & $0.40 \pm 0.08$ & \\
\hline Large FOV with slice thickness $=5 \mathrm{~mm}$ & $0.40 \pm 0.06$ & \\
\hline
\end{tabular}

Results are expressed as the mean \pm standard deviation

* $: \mathrm{P}<0.05$

** $: \mathrm{P}<0.01$ 
TABLE 3.

Visual image quality for the four parameter combinations

$\left.\begin{array}{cccc}\hline \text { Group } & \multicolumn{1}{c}{\text { Artifact }} & \text { Seminal vesicle } & \text { Overall image } \\ \hline \text { Small FOV with slice thickness }=2 \mathrm{~mm} & 1.67 \pm 0.82 & 2.13 \pm 1.06 & 2.33 \pm 1.05 \\ \text { Small FOV with slice thickness }=5 \mathrm{~mm} & 1.27 \pm 0.46 & 1.73 \pm 0.59 & 2.00 \pm 0.65] * \\ \text { Large FOV with slice thickness }=2 \mathrm{~mm} & 1.20 \pm 0.41 & 1.40 \pm 0.5 & 1.20 \pm 0.41]]_{* *} \\ \text { Large FOV with slice thickness }=5 \mathrm{~mm} & 1.47 \pm 0.74 & 2.07 \pm 0.80 & 2.33 \pm 0.49\end{array}\right]$

Results are expressed as the mean \pm standard deviation

$* \quad: \mathrm{P}<0.05$

$* *: \mathrm{P}<0.01$

$* * *: \mathrm{P}<0.001$
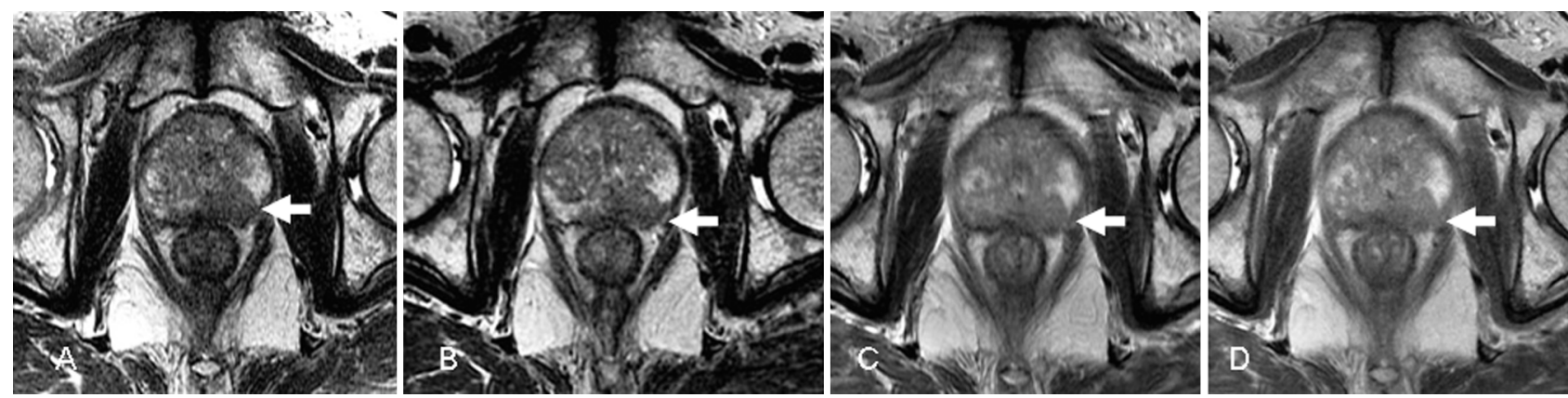

Fig. 1. T2WI using a cardiac coil of a 74-year-old man with prostate cancer (arrow). A: slice thickness 2 mm with small FOV, B: slice thickness $2 \mathrm{~mm}$ with large FOV, C: slice thickness $5 \mathrm{~mm}$ with small FOV, and D: slice thickness $5 \mathrm{~mm}$ with large FOV. A and B show increased spatial resolution compared to C and D (e.g., capsule delineation, visibility of central gland, edge of muscle and bone), but the overall image quality of A is inferior to B. The overall image quality of B is superior to the other three images.

We observed minor and moderate artifacts caused by motion of the abdominal wall, but the differences between the 4 parameter combinations were not statistically significant. We did not observe any other types of artifacts in this study. We found no significant differences in the image quality of the seminal vesicle among the 4 parameter combinations.

Concerning the quality of the overall image, although differences between the images obtained at slice thicknesses of $2 \mathrm{~mm}$ and $5 \mathrm{~mm}$ with a small FOV were not statistically significant, overall image quality obtained at a slice thickness of $2 \mathrm{~mm}$ with a large FOV was significantly superior to the images obtained at a slice thickness of $2 \mathrm{~mm}$ with a small FOV $(P<0.01)$, slice thickness of $5 \mathrm{~mm}$ with a small FOV $(P<0.05)$, and slice thickness of $5 \mathrm{~mm}$ with a large FOV $(P<0.001)$ (Table 3, Fig. 1).

\section{DISCUSSION}

MR pulse sequences with high spatial resolution are required to assess tumor margins correctly and to detect small cancerous areas. The low SNR of conven- tional 1.5T MRI using body coils has driven the development of endorectal coils, recently used in combination with pelvic-array coils for high spatial resolution in prostate cancer [13].

3T MRI has a nearly two-fold higher SNR compared to conventional 1.5T MRI and provides some advantages for clinical MRI, such as faster scan time and better spatial resolution. In terms of local staging, 3T MRI using the phased-array coil can be expected to offer good image quality, equivalent to endorectal 1.5T MRI. Sosna et al. [5] reported that prostate 3T MRI quality with large FOV using an external phasedarray coil was similar to that of endorectal 1.5T MRI for evaluating the posterior border, seminal vesicles, and neurovascular bundles. In addition, Park et al. [6] recently reported that $3 \mathrm{~T}$ MRI using a phased-array coil was equivalent to $1.5 \mathrm{~T}$ MRI using endorectal coil for evaluating local staging accuracy without a significant loss of image quality.

Heijmink et al. [14] found that image quality and accuracy of prostate cancer localization and staging at 3T MRI using an endorectal coil were better than at 3T MRI using a body-array coil. While the endorectal coil 
is superior to the body phased-array coil when used at the same standard clinical field strength, it has some limitations for use. The use of an endorectal coil is contraindicated in patients examined shortly after pelvic surgery and radiation therapy [15]. Moreover, its use may also deform the peripheral zone [16], resulting in signal hyperintensity near the rectum, peripheral zone, and neurovascular bundles and difficult image interpretation. Because there are the limitations associated with use of the endorectal coil and the fact that the endorectal coil is more invasive than the phased-array coil, developing methods of achieving good image quality of 3T MRI with a phased-array coil is of urgent importance.

FOV and slice thickness affect SNR, contrast and spatial resolution of the MR image. Spatial resolution increases as slice thickness and FOV decrease, but SNR is decreased. It is important to determine the optimal FOV and slice thickness for 3T MRI of the prostate. We designed our study to clarify optimal prostate image quality of T2WI obtained with 3T MRI using a cardiac array or torso array coil and 4 combinations of FOV and slice thickness. In this study, we considered scan time to be one of the most important factors in MRI examinations. Therefore we set each parameter so that the SNR could be evaluated for each FOV and slice thickness within a clinically acceptable 5-minute scan time. As a result, TR/TE, the matrix and bandwidth of each image protocol are different. A slice thickness of $2 \mathrm{~mm}$ is difficult to achieve with 1.5T MRI, while a $5 \mathrm{~mm}$ thickness is common. The large FOV used in this study $(26 \times 26 \mathrm{~cm})$ is common, and the smallest FOV image size that does not result in wraparound artifacts is $18 \times 18 \mathrm{~cm}$.

The cardiac array coil has a superior SNR compared to the torso array coil. Therefore, 13 of the 15 patients were examined using an 8-channel cardiac array coil.

Prostate cancer in the peripheral zone and transitional zone is darker than normal tissue on T2WI. Therefore, tissue contrast in images of the prostate is one of the most important diagnostic factors. Although the contrast between prostate cancer and the peripheral zone is ideal, it is difficult to assess ROI in peripheral zones compressed by a central gland enlarged due to age-related anatomical changes. Therefore, we analyzed the contrast between prostate cancer and muscle. Morakkabati-Spitz et al [12] reported the contrast between muscle and solid tumor in the pelvis at 3T MRI. So, we suspected that our contrast data was adequate. Because an increase of $21 \%$ in the $\mathrm{T} 1$ relaxation time and a decrease of $16 \%$ in the $\mathrm{T} 2$ relaxation time in the prostate have been observed in 3T MRI compared to 1.5T MRI [17], we used longer TR and TE times to increase image contrast for the images obtained in this study. Although the contrast of the image obtained at a slice thickness of $2 \mathrm{~mm}$ with a small FOV was inferior to the other parameter combinations, the image obtained at a slice thickness of $2 \mathrm{~mm}$ with a large FOV showed no statistically significant difference in contrast when compared to the images obtained at a slice thickness of $5 \mathrm{~mm}$. We suggest that the slightly larger voxel size in the large FOV studies can offer a contrast advantage.

In this study, we observed only minor and moderate artifacts due to motion. The application of an intramuscular injection of glucagon and placement of a dielectric pad in front of the body successfully minimized motion and susceptibility artifacts. To obtain images with high SNR, we used a slice thickness of 2 $\mathrm{mm}$, which required a narrow bandwidth. We were aware that a narrower bandwidth might intensify chemical shift artifacts, but fortunately we did not observe this. We suspect that chemical shift artifacts deceased due to the thinner slice thickness. To decrease image scan time, we increased the echo train length (ETL). We were aware that any increase in the ETL might intensify blurring artifacts, but we did not observe such an increase in our study.

Although Sosna et al. [5] reported that the image quality of the seminal vesicle with a large FOV was significantly superior to that of a small FOV, we found no significant difference in our study. However, image quality of the seminal vesicle at a slice thickness of 2 $\mathrm{mm}$ tended to be higher in the large FOV than in the small FOV. On the other hand, in images obtained at a slice thickness of $5 \mathrm{~mm}$, image quality of the seminal vesicle was lower in the large FOV than in the small FOV. We suspect that this result can be explained by an inferior SNR at a slice thickness of $5 \mathrm{~mm}$ with a large FOV compared to that of other images. This is most likely due to the smaller voxel size and the wider bandwidth compared to other images, given the similar scan times.

The voxel size of the image obtained at a slice thickness of $2 \mathrm{~mm}$ was smaller than that at a slice thickness of $5 \mathrm{~mm}$, so the SNR of the image obtained at a slice thickness of $2 \mathrm{~mm}$ was inferior to that at a slice thickness of $5 \mathrm{~mm}$. And concerning tissue contrast, there was no statistically significant difference between the images obtained at slice thicknesses of $2 \mathrm{~mm}$ and $5 \mathrm{~mm}$ with large FOV. However, the overall image quality obtained at a slice thickness of $2 \mathrm{~mm}$ with a large FOV was higher than that at a slice thickness of 
$5 \mathrm{~mm}$. The thinner slice can offer higher spatial resolution and reduce the partial volume effect while the slightly larger voxel size in the large FOV will probably offer a SNR advantage. This may help explain the increase in image quality. The overall image and the periprostatic structures are superbly depicted on T2WI. There have been several recent reports in the literature dealing with the use of diffusion-weighted (DW) imaging [18,19] and 1H-MR spectroscopic imaging $[20,21]$ for prostate cancer. Both imaging techniques are useful for identifying prostate cancer, however, they are not suitable for the determination of tumor spread due to their low resolution. High resolution T2WI has proven one of the most useful methods to identify tumors in the prostate and their spread into the adjacent periprostatic tissues because of the excellent anatomic images that can be obtained of the gland.

In principle, a higher signal allows an increase in the spatial resolution. However, SAR limits are reached earlier at a high magnetic field. Parallel imaging techniques reduce the energy deposition, ETL, scan time and presence of blurring artifacts. We used parallel imaging for the large FOV, but could not use it with the small FOV. Unfortunately, our high magnetic field cannot use parallel imaging when the FOV is less than $20 \mathrm{~cm}$. This is one limitation of our study. Our study had four additional limitations. First, because data analysis was performed in consensus, we cannot provide data on interobserver variability. Second, our study included only a small patient population. Third, the MR images were not compared with the results of histological mapping of the prostate. Although the locations of all the lesions were consistent with estimates obtained by TRUS-guided needle biopsy, biopsy results might differ from those of a radical prostectomy with respect to tumor localization. Fourth, the imaging protocols were not uniform. Given the importance of scan time, using similar scan times for each protocol is necessary. In future, it will be necessary to compare 3T MRI with 1.5T MRI in patients undergoing radical prostectomy.

In conclusion, the image obtained at a slice thickness of $2 \mathrm{~mm}$ with a small FOV was inferior to the images obtained with the same slice thickness and a large FOV as well as to images obtained at a slice thickness of $5 \mathrm{~mm}$. However, in the case of large FOV, we found no statistically significant difference in contrast between images at a slice thickness of $2 \mathrm{~mm}$ and those at a slice thickness of $5 \mathrm{~mm}$. Overall image quality at a slice thickness of $2 \mathrm{~mm}$ with a large FOV was superior to that of other images because the thin slice offers a higher spatial resolution. Images obtained with a small FOV and thin slice thickness are theoretically ideal, however, the best images were obtained with a large FOV and slice thickness of $2 \mathrm{~mm}$. Although the imaging protocols used in this study will improve with the development of 3T MRI coils and software, our data suggest that high spatial resolution images obtained with 3T MRI can provide more clinically relevant information in an acceptable scan time than with 1.5T MRI.

\section{REFERENCES}

1. Scarabino T, Nemore F, Giannatempo GM, Bertolio A, Di Salle F et al. 3.0 T magnetic resonance in neuroradiology. Eur J Radiol 2003; 48:154-164.

2. Berg A, Singer T, and Moser E. High-resolution diffusivity imaging at 3.0 $\mathrm{T}$ for the detection of degenerative changes: a trypsin-based arthritis model. Invest Radiol 2003; 38:460-466.

3. Chen W, and Ugurbil K. High spatial resolution functional magnetic resonance imaging at very-high-magnetic field. Top Magn Reson Imaging 1999; 10:63-78.

4. Yoneka Y, Kwee 1L, Fujii Y, and Nakada T. Criteria for normal cavities observed within the adult hippocampus: high resolution magnetic resonance imaging study on a 3.0 T-system. J Neuroimaging 2002; 12:231-235.

5. Sosna J, Pedrosa I, Dewolf WC, Mahallati H, Lenkinski RE et al. MR imaging of the prostate at 3 Tesla: comparison of an external phased-array coil to imaging with an endorectal coil at 1.5T Tesla. Acad Radiol 2004; 11:857862.

6. Park BK, Kim B, Kim CK, Lee HM, and Kwon GY. Comparison of phased-array 3.0T and endorectal $1.5 \mathrm{~T}$ MRI in the evaluation of local staging accuracy for prostate cancer. J Comput Assist Tomogr 2007; 31:534-538.

7. Busse RF. Reduced RF power without blurring: correcting for modulation of refocusing flip angle in FSE sequences. Magen Reson Med 2004; 51:1031-1037.

8. Wansapura JP, Holand SK, Dunn RS, and Ball WS Jr. NMR relaxation times in human brain at 3.0 T. J Magn Reson Imaging 1999; 9:531-538.

9. Al Kwifi O, Emery DJ, and Wilman AH. Vessel contrast at three tesla in time-of-flight magnetic resonance angiography of the intracranial and carotid arteries. Magn Reson Imaging 2002; 20:181-187.

10. Thomas SD, Al Kwifi O, Emery DJ, and Wilman AH. Application of magnetization transfer at $3.0 \mathrm{~T}$ in threedimensional time-of-flight magnetic resonance angiography of the intracranial arteries. J Magn Reson imaging 2002; 15:479-483.

11. von Falkenhausen M, Gieseke J, Morakkabati N, Lutterbey G, Blömer R et al. 3T MRI of the liver after SPIO application. A comparison to 1.5T. Proc Int Soc 2004; 11:904.

12. Morakkabati-Spitz N, Gieseke J, Kuhl C, Lutterbey G, von Falkenhausen $\mathrm{M}$ et al. MRI of the pelvis at 3T: very high spatial. Eur Radiol 2006; 16:634-641.

13. Bydder M, Larkman DJ, and Hajnal JV. Combination of signals from array coils using image-based estimation of coil sensitivity profiles. Magn Reson Med 2002; 47:539- 
548.

14. Heijmink SW, Fütterer JJ, Hambrock T, Takahasi S, Scheenen TW et al. Prostate cancer: body-array versus endorectal coil MR imaging at 3T-comparison of image quality, localization, and staging performance. Radiology 2007; 244:184-195.

15. Maio A, and Rifkin MD. Magnetic resonance imaging of prostate cancer: update. Top Magn Reson Imaging. 1995; 7:54-68.

16. Hirose M, Bharatha H, Hata N, Zou KH, Warfield SK et al. Quantitative MR imaging assessment of prostate gland deformity before and during MR imaging-guided brachytherapy. Acad Radiol 2002; 9:906-912.

17. De Bazelaire CMJ, Duhamel GD, Rofsky NM, and Alsop DC. MR imaging relaxation times of abdominal and pelvic tissues measured in vivo at $3.0 \mathrm{~T}$ : preliminary results. Radiology 2004; 230:652-659.

18. Shimofusa R, Fujimoto H, Akamata H, Motoori K, Yamamoto $\mathrm{S}$ et al. Diffusion-weighted imaging of prostate cancer. J Comput Assist Tomogr 2005; 29:149-153.

19. Gibbs P, Pickles MD, and Turnbull LW. Diffusion imaging of the prostate at 3.0 tesla. Invest Radiol 2006; 41:185-188.

20. Fütterer JJ, Heijmink SW, Scheenen TW, Veltman J, Huisman $\mathrm{HJ}$ et al. Prostate cancer localization with dynamic contrast-enhanced MR imaging and proton MR spectroscopic imaging. Radiology 2006; 241:449-458.

21. Reinsberg SA, Payne GS, Riches SF, Ashley S, Brewster JM et al. Combined use of diffusion-weighted MRI and ${ }^{1} \mathrm{H}$ MR spectroscopy to increase accuracy in prostate cancer detection. AJR Am J Roentgenol 2007; 188:91-98. 